\title{
A study of the efficacy and tolerability of capecitabine and lobaplatin in advanced HER-2 negative breast cancer patients
}

\author{
Yuan Yuan $^{1} \wedge$, Lili Zhang ${ }^{1 \wedge}$, Zhe Zhang $^{2}$, Yu Qian $^{3} \wedge$, Yue Teng $^{1}$ \\ ${ }^{1}$ Department of Chemotherapy, Jiangsu Cancer Hospital \& Jiangsu Institute of Cancer Research \& The Affiliated Cancer Hospital of Nanjing \\ Medical University, Nanjing, China; ${ }^{2}$ Department of Pathology, Jiangsu Cancer Hospital \& Jiangsu Institute of Cancer Research \& The Affiliated \\ Cancer Hospital of Nanjing Medical University, Nanjing, China; ${ }^{3}$ Department of Oncology, The Affiliated Cancer Hospital of Nanjing Medical \\ University, Nanjing, China \\ Contributions: (I) Conception and design: L Zhang; (II) Administrative support: Y Yuan; (III) Provision of study materials or patients: Y Yuan; (IV) \\ Collection and assembly of data: Y Teng; (V) Data analysis and interpretation: Z Zhang, Y Qian; (VI) Manuscript writing: All authors; (VII) Final \\ approval of manuscript: All authors. \\ Correspondence to: Lili Zhang, MD. Department of Chemotherapy, Jiangsu Cancer Hospital \& Jiangsu Institute of Cancer Research \& The Affiliated \\ Cancer Hospital of Nanjing Medical University, No. 42 Baiziting, Nanjing 210009, China. Email: longrenyu@sina.com.
}

\begin{abstract}
Background: This study sought to examine the efficacy and adverse reactions of capecitabine and lobaplatin in the treatment of metastatic human epidermal growth factor receptor 2 (HER-2) negative breast cancer (BC).

Methods: This retrospective study examined 45 patients diagnosed with advanced HER-2 negative BC. Patients were enrolled in this study from November 2015 to June 2019. The patients received capecitabine and lobaplatin combination therapy. The therapeutic efficacy and side effects were evaluated after at least 2 cycles of treatment.
\end{abstract}

Results: Therapeutic efficacy and adverse reactions were evaluated in 38 patients, comprising 12 cases of partial response (PR), 19 cases of stable disease (SD), and 7 cases of progressive disease (PD). Among these, 3 patients required treatment delays or dose reductions for subsequent cycles, and 2 patients discontinued treatment. The overall response rate (ORR) was $31.58 \%$ and the disease control rate (DCR) was $81.58 \%$. The ORR and DCR for hormone receptor positive, HER-2 negative (HR+/HER-2-) and triple negative breast cancer (TNBC) patients were $31.82 \%$ and $31.25 \%$, and $86.36 \%$ and $75 \%$, respectively. The median progression free survival (PFS) was 8 months, 6 months, and 6 months in patients receiving the therapeutics as a first-line, second-line, or third-line and beyond treatment, respectively. The main side effects were myelosuppression, including granulocytopenia, thrombocytopenia, and anemia. Among patients with grade 1 side effects or above, 28 patients (73.68\%) had myelosuppression, and 13 patients $(34.21 \%)$ had gastrointestinal reactions. Further, we investigated the association between side effects and clinical outcomes, and found that PFS was increased in patients with myelosuppression and gastrointestinal reactions.

Conclusions: Capecitabine and lobaplatin combination therapy was effective and well tolerated among patients with advanced HER-2 negative BC.

Keywords: Advanced breast cancer; lobaplatin; capecitabine; side effects; HER-2 negative

Submitted Apr 30, 2021. Accepted for publication Jul 07, 2021.

doi: $10.21037 / \mathrm{atm}-21-2702$

View this article at: https://dx.doi.org/10.21037/atm-21-2702

^ ORCID: Yuan Yuan, 0000-0002-2060-5969; Lili Zhang, 0000-0002-4646-6565; Yu Qian, 0000-0002-6348-9638. 


\section{Introduction}

Breast cancer (BC) ranks number one among malignant tumors in females (1). It can be divided into several different molecular subtypes, including the luminal subtype, triple negative breast cancer (TNBC), and human epidermal growth factor receptor 2 (HER-2) positive BC depending on the status of the estrogen receptor (ER), progesterone receptor (PR), and HER-2 (2). It has been reported that $72.7 \%$ breast cancers were hormone receptor-positive/ HER2-negative, and $12.2 \%$ were triple-negative (hormone receptor-negative/HER2-negative), and 14.1\% HER2positive with either positive or negative hormone receptor, therefore, the breast cancer patients with HER2-negative breast cancer were about $85.9 \%$.

Two main types of anti-HER-2 medicines can be used to treat HER-2 positive metastatic breast cancer (MBC), including anti-HER2 antibodies, such as pertuzumab, trastuzumab, and T-DM1, and small molecule tyrosine kinase inhibitors, such as lapatinib, pyrotinib, and neratinib $(3,4)$. However, chemotherapy remains the basic treatment for advanced MBC, especially for HR+/HER-2- MBC patients with endocrine resistance, rapid progression, and a visceral crisis.

Nearly all MBC patients with adjuvant treatment options will receive the combination therapy of anthracyclines and taxanes (paclitaxel and docetaxel). However, some patients who have a recurrence after adjuvant treatment, may have developed a resistance to these two kinds of drugs. There is a lack of alternative options for patients who have a recurrence after treatment with anthracyclines and taxanes (5). In clinical practice, a combination of drugs with different mechanisms is usually used to achieve favorable therapeutic effects in MBC. Platinum-based medications, such as the first-generation cisplatin and the second-generation carboplatin, are the main choice of medicines (6). However, their application is usually limited due to severe gastrointestinal adverse reactions, renal toxicity, neurotoxicity, ototoxicity, and significant myelosuppression.

Lobaplatin is a third-generation platinum anti-cancer drug, and has shown strong anti-cancer activity and low toxicity (7). Lobaplatin has been shown to have an obvious efficacy on various tumors, such as BC, lung cancer and hepatocellular carcinoma in China (8-10), and has been approved for the treatment of BC, small cell lung cancer, and chronic myelogenous leukemia. There is no standard treatment for recurrent or metastatic $\mathrm{BC}$ with negative HER-2 BC patients who had been pretreated with both anthracyclines and taxanes. However, there is a great need to develop reasonable treatment plans to obtain the maximum clinical efficacy and avoid intolerable side effects. Capecitabine is a chemotherapy medication often used to treat breast cancer as monotherapy or used together with docetaxel. Common side effects include abdominal pain, vomiting, diarrhea, weakness, and rashes.

Both capecitabine and lobaplatin have been used in breast cancer patients, however, little is known about the efficacy and tolerability of the combination treatment of capecitabine with lobaplatin in breast cancer patients. For patients with HER2 negative advanced breast cancer that are resistant to anthracycline and taxane treatment, the treatment plan usually contains capecitabine. In this study, we reported that the combination of capecitabine and lobaplatin treatment demonstrated good efficacy and safety profile. Furthermore, capecitabine can continuously be used as the maintenance treatment after completion of the combination therapy in patients with advanced triplenegative breast cancer and endocrine resistant HR+/HER2 negative breast cancer.

We present the following article in accordance with the STROBE reporting checklist (available at https://dx.doi. org/10.21037/atm-21-2702).

\section{Methods}

\section{General information}

Total of 45 recurrent MBC patients (with an age range 40-70 years, and a median age of 53) who received a combination therapy of lobaplatin and capecitabine at the Jiangsu Cancer Hospital from November 2015 to June 2019 were retrospectively analyzed in this study, and the therapeutic effects of and adverse reactions to this therapy were observed. Among these patients, 7 discontinued the treatment without efficacy evaluations for personal reasons. The remaining 38 patients, who received at least 2 cycles of treatments, underwent a computed tomography (CT) scan after every 2 cycles of treatments to evaluate the therapeutic effects. Patients with recurrent luminal subtype (ER or/and PR+, HER-2-) in this study had either a primary endocrine resistance or visceral crisis or received adjuvant therapy of anthracyclines and taxanes before enrolling in this study. The Eastern Cooperative Oncology Group (ECOG) scores 
Table 1 Baseline patient characteristics $(n=38)$

\begin{tabular}{lc}
\hline Group & $\mathrm{n}(\%)$ \\
\hline Age (years) & $27(71.05)$ \\
$<60$ & $11(28.95)$ \\
$\geq 60$ & \\
Treatment line & $11(28.95)$ \\
1 & $14(36.84)$ \\
2 & $13(34.21)$ \\
$\geq 3$ & \\
Molecular subtyping & $22(57.89)$ \\
HR+/HER-2- & $16(42.11)$ \\
TNBC & \\
Location of metastatic lesions & $28(73.68)$ \\
Visceral locations & $10(26.32)$ \\
Non-visceral locations & \\
\hline
\end{tabular}

TNBC, triple negative breast cancer.

of patients ranged from 0 to 1 (see Table 1).

\section{Study methods}

We have selected the dose according to the drug instructions and other clinical research results. Capecitabine was administered by $1,000 \mathrm{mg} / \mathrm{m}^{2}$ twice a day from day 1 to day 14. Lobaplatin was administered by $30 \mathrm{mg} / \mathrm{m}^{2}$ daily for 3 weeks as 1 cycle, and repeated CT scans were performed every 2 cycles to evaluate the therapeutic effects. Patients who suffered with III-IV grade side effects and intolerance during the treatments had their doses adjusted. A blood test was performed before each cycle of treatment to evaluate cell counts of neutrophils and platelets, and liver and kidney function. All treated patients underwent among 1-10 cycles (median cycle: 6) of the combination therapy, followed by maintenance therapy with capecitabine. All procedures performed in this study involving human participants were in accordance with the Declaration of Helsinki (as revised in 2013). The study was approved by ethics board of Jiangsu Cancer Hospital (No. 2020-041). Individual consent for this retrospective analysis was waived.

\section{Clinical follow-up}

The evaluation of the efficacy effects was based on the
RECIST 1.1 (2009) (11). Repeated imaging and an evaluation of the therapeutic effects were performed every 2 cycles. Complete response (CR) refers to conditions for which all the target foci disappeared; PR refers to conditions with a total length reduction of $\geq 30 \%$ for the baseline foci; progression of disease (PD) refers to conditions with a total length increase of $\geq 20 \%$ for the baseline foci or new foci(s); stable disease (SD) refers to conditions between PD and PR. Patients who showed no disease progression continued with the current treatment regimens, while an alternative treatment was provided to patients who showed disease progression.

The evaluation of the therapeutic effects included the objective response rate (ORR), disease control rate (DCR), and progression free survival (PFS). PFS was defined from the first day of treatment to disease progression or death from any cause.

\section{Adverse reactions}

All the patients underwent toxicity assessments. Blood tests were performed before and after every chemotherapy cycle or as necessary. The side effects were evaluated according to the National Cancer Institute Common Toxicity Criteria for Adverse Events (NCI CTCAE) as Grades 0-IV.

\section{Statistical analysis}

SAS 9.3 statistical software and Graphpad Prism 8 were used for the statistical analysis. The Kaplan-Meier method was used in the survival analysis. A $\chi^{2}$ test or Fisher's exact test was used to assess differences in the subgroups. A $\mathrm{P}<0.05$ indicated a statistically significant difference.

\section{Results}

\section{Clinical activity}

All patients received at least 1 cycle of a combination treatment. The number of cycles patients received ranged from 1 to 10 , and the median period was 5 months. Following the combination treatment, patients received maintained capecitabine treatment until disease progression. Of the patients, 7 received only 1 cycle of treatment and dropped out due to personal reasons without efficacy evaluations. The remaining 38 patients had 2 or more metastatic sites, and 28 patients $(73.68 \%)$ had visceral involvement, with liver as the most frequent visceral 
Table 2 ORR in the different subgroups

\begin{tabular}{lccc}
\hline Group & $\mathrm{n}$ & ORR (\%) & $\chi^{2}$ \\
\hline Treatment line & 11 & & 2.6689 \\
1 & 14 & 45.45 & 0.2633 \\
2 & 13 & 35.71 & 15.38 \\
$\geq 3$ & & & 0.0014 \\
Molecular subtyping & 22 & 31.82 & 0.9703 \\
HR+, HER-2- & 16 & 31.25 & 0.8421 \\
TNBC & & & 0.3588 \\
Location of metastatic lesions & 28 & 35.71 & 20.00 \\
Visceral locations & 10 & & \\
Non-visceral locations & & & \\
\hline
\end{tabular}

ORR, overall response rate; TNBC, triple negative breast cancer.

Table 3 DCR in different subgroups

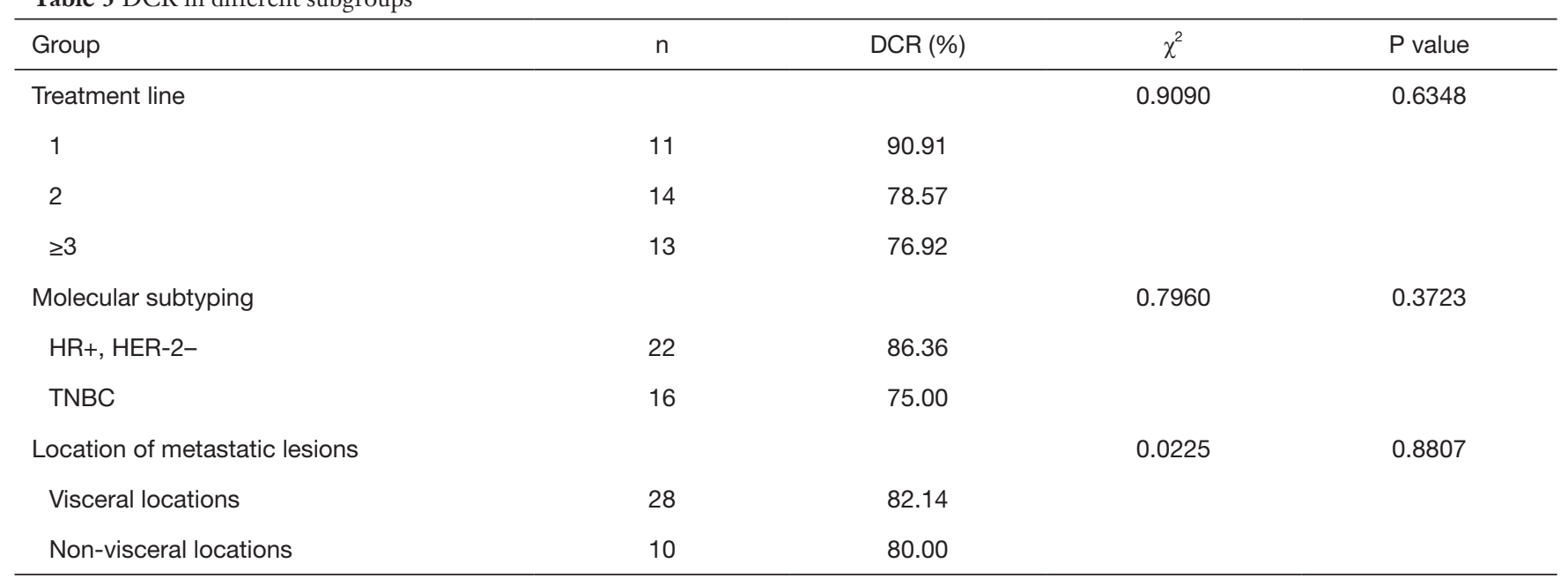

$\mathrm{DCR}$, disease control rate; TNBC, triple negative breast cancer.

metastatic site. For these patients, 2 could not tolerate the adverse reactions after 2 cycles of treatments, which led to treatment interruption. Of these 2 patients, 1 patient had severe anemia and the other had grade 4 thrombocytopenia twice. Both patients were provided with alternative therapeutic plans.

\section{Clinical response}

Among these cases, there was no case of CR; however, there were 12 cases of PR, 19 cases of SD, and 7 cases of PD. The total overall response rate (ORR) was $31.58 \%$, while the DCR was $81.58 \%$. In the subgroup analysis, the ORRs and DCRs were $31.82 \%$ and $86.36 \%$ in the HR+/HER-2subgroup and $31.25 \%$ and $75 \%$ in the TNBC subgroup, respectively. The ORR and DCR were not significantly different between these two subgroups (see Tables 2,3).

\section{Clinical benefits}

All 38 patients were eligible for the PFS analysis. At the time of the analysis (January 2020), the median PFS was 6.5 months (ranging from 1.5 to 34 months), and there was no difference between the subgroups regardless of 
Table 4 PFS in different subgroups

\begin{tabular}{lccc}
\hline Group & $\mathrm{n}$ & $\mathrm{mPFS}(95 \% \mathrm{Cl})$ (month) & $\chi^{2}$ \\
\hline Treatment line & 11 & 8.1504 & 0.1425 \\
1 & 14 & $6(4,9.5)$ & \\
2 & 13 & $6(2,8)$ & 0.0015 \\
$\geq 3$ & & & 0.9695 \\
Molecular subtyping & 22 & $6(3,10)$ & 0.1357 \\
HR+, HER-2- & 16 & $7(4,9)$ & 0.7126 \\
TNBC & & $6(3,10)$ & \\
Metastatic sites & 28 & $7(6,10)$ & \\
Visceral sites & 10 & & \\
Non-visceral sites & & & \\
\hline
\end{tabular}

mPFS, median progression free survival; Cl, confidence interval; TNBC, triple negative breast cancer.

treatment lines, molecular types, and visceral metastasis status (see Table 4).

In patients receiving the combination therapy as the first-line treatment, all had TNBC except 1 patient who had $\mathrm{HR}+$ with a visceral crisis. The median PFS of the patients with TNBC was 7.5 months [95\% confidence interval (CI): 2, 10 months]. For patients receiving the combination therapy as the second-line treatment, the median PFS was 6.5 months (95\% CI: 3, 11 months) and 4 months (95\% CI: 2, 9 months) for the HR+ and TNBC subgroups respectively. For patients receiving the treatment as a thirdline treatment or above, all the patients had $\mathrm{HR}+\mathrm{BC}$ and a median PFS of 6 months (95\% CI: 2, 8 months). We have presented the CT scan results from one representative patient to demonstrate the efficacy of the combination therapy. As shown in the CT scan, the metastatic lesions in the liver have shrunk over time and the patient has partial response to the combination therapy, in Figure 1.

\section{Side effects}

In general, the regime was well tolerated, and the side effects were manageable for all except 2 patients. Of these 2 patients, one patient developed serious anemia and the other had grade IV thrombocytopenia twice with a long recovery time. Thus, alternative therapies were provided to these 2 patients. Three patients encountered grade III platelets reduction twice, or grade IV thrombocytopenia once; thus, their lobaplatin doses were reduced (see Table 5).
The other common side effect was gastrointestinal reaction. Thirteen patients had episodes of anorexia, nausea, and/or vomiting, which were manageable and tolerated. Other side effects observed included hand-foot syndrome for 1 patient, and mild fatigue in 4 patients.

The grade 1 side effects improved in all cases without intervention and had no effect on subsequent drug administration. Conversely, patients with side effects $\geq$ grade 2 required treatments with granulocyte colony-stimulating factor for neutropenia and platelet transfusion for thrombocytopenia. No toxicity-related deaths were observed during treatment.

\section{The relationship between side effects and prognosis}

We also investigated the relationship between side effects and clinical outcomes. The Kaplan-Meier method was used to estimate PFS for 28 patients with grade 1 or worse myelosuppression, including neutropenia and thrombocytopenia was compared to lacking myelosuppression, the median PFS was 7 and 5 months, respectively ( $\mathrm{P}=0.0255$; Figure $2 A)$. Similarly, the 13 patients with grade 1 or higher gastrointestinal reactions had significantly longer PFS compared to absent gastrointestinal reactions; the median PFS was 9 and 7 months, respectively $(\mathrm{P}=0.0150$; see Figure 2B).

\section{Discussion}

Chemotherapy is the main choice of therapy for advanced 
2018-05

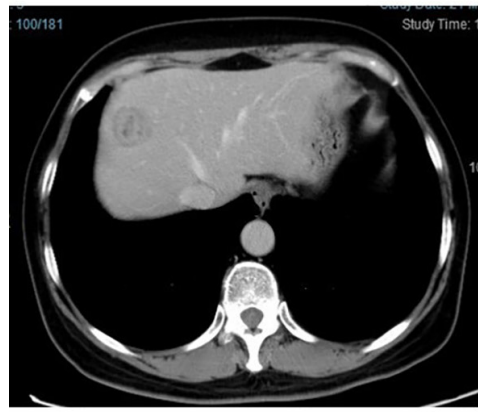

2018-12

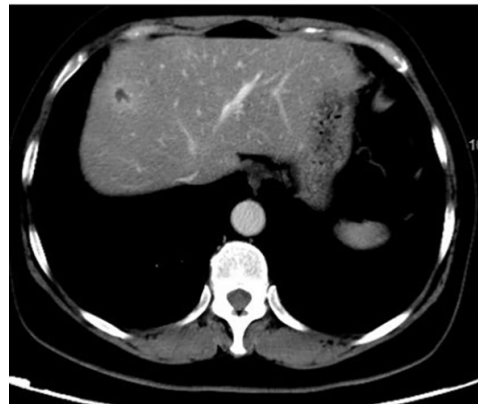

2018-07

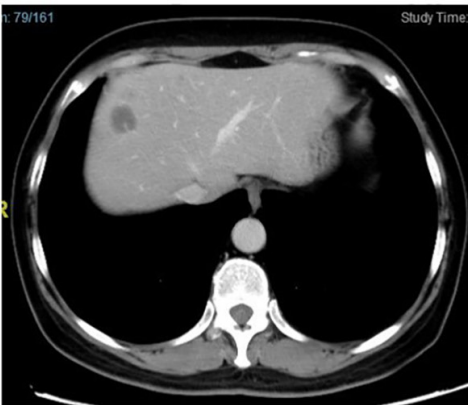

2019-02

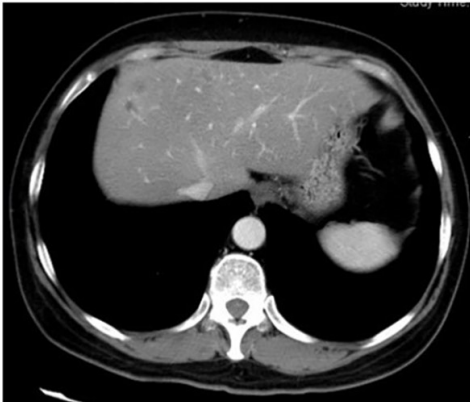

2018-09

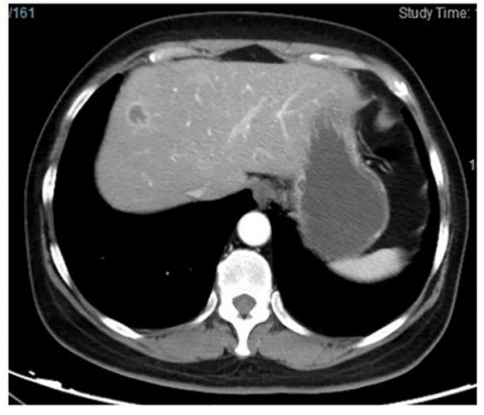

2019-04

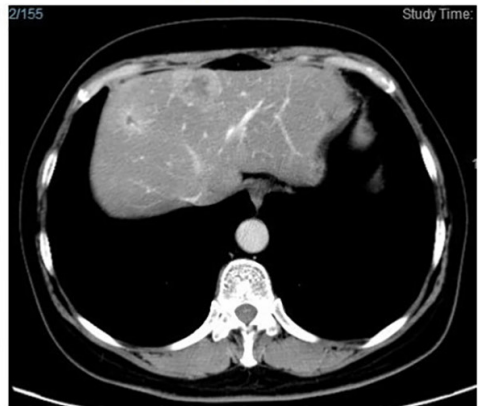

Figure 1 The CT scan of one representative patient during the treatment of capecitabine and lobaplatin in combination. CT, computed tomography.

Table 5 Side effects in the treatment of metastatic breast cancer patients

\begin{tabular}{lc}
\hline Toxicities & $\mathrm{n}(\%)$ \\
\hline Myelosuppression & 28 \\
Grade I & $1(2.63)$ \\
Grade II & $15(39.47)$ \\
Grade III & $9(23.69)$ \\
Grade IV & $3(7.89)$ \\
Gastrointestinal reaction & 13 \\
Grade I & $4(10.53)$ \\
Grade II & $5(13.16)$ \\
Grade III & $4(10.53)$ \\
Others & 5 \\
Hand-foot syndrome & $1(2.63)$ \\
Fatigue & $4(10.53)$ \\
\hline
\end{tabular}

A

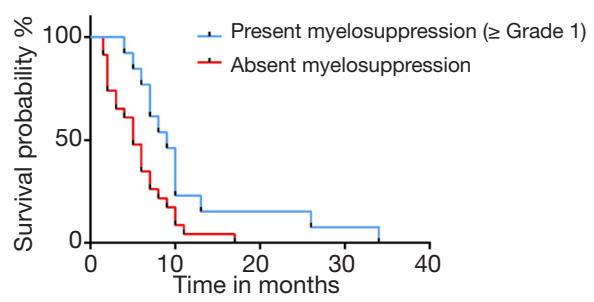

B

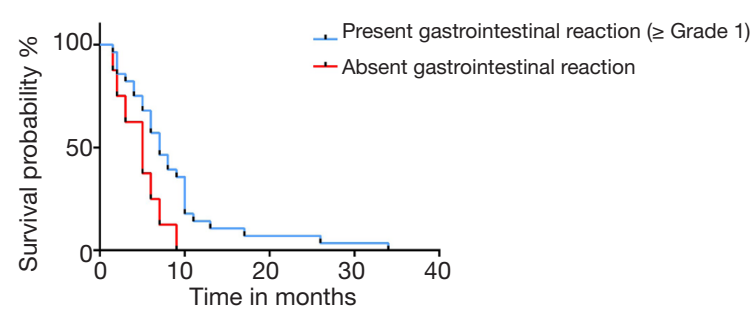

Figure 2 The relationship between PFS and side effects. (A) The relation between PFS and myelosuppression; (B) the relation between PFS and gastrointestinal reaction. PFS, progression free survival. 
TNBC and HR+/HER-2-MBC patients who are resistant to endocrine therapy, have rapid progressing visceral crises, or a high tumor burden and metastasis. However, there is no standard chemotherapy for MBC patients, especially those in the late stage of the disease. The identification of an effective chemotherapeutic combination represents one of the major challenges in the palliative treatment of HER-2 negative MBC (12).

The identification of reasonable regimes that have maximal therapeutic efficacy and minimal side effects requires further study and investigation. Platinum-based chemotherapy has been proven to improve the major clinical outcomes among MBC patients $(13,14)$. Lobaplatin, a third-generation platinum anti-cancer drug, is superior to cisplatin in terms of its efficacy and better tolerance in MBC patients (15). Lobaplatin-based regimen chemotherapy for MBC patients is effective and well tolerated (16).

Capecitabine is an oral fluoropyridine derivate widely used in MBC, which is also commonly used in combination regimens with other chemotherapeutic agents in anthracycline and taxane-resistant patients. Further, capecitabine can also be used as a monotherapy for maintenance therapy in $\mathrm{MBC}$, especially in endocrine resistant and TNBC patients (17-19).

There is currently no standard treatment for patients with triple-negative breast cancer and endocrine-resistant HER-2 negative breast cancer who have failed previous treatment with anthracyclines and taxanes. Capecitabine and platinum drugs can be used for those patients. Compared with the first-generation platinum drugs, the thirdgeneration platinum drugs including lobaplatin developed in China have less nephrotoxicity and gastrointestinal reactions. Therefore, the treatment plan of capecitabine combined with lobaplatin was used, and capecitabine can further be selected as the maintenance treatment after the combination therapy. In the present study, we explored the efficiency and side effects of the combination therapy of lobaplatin and capecitabine in HER-2 negative MBC patients.

Patients enrolled in this study, who all had MBC and received combination therapy as a first-line, second-line or third-line or above treatment, had a median PFS of 8 months, 6 months, and 6 months, respectively. Nearly $1 / 3$ of the patients enrolled in this study had the therapy as a third-line or above therapy. The ORR and DCR were $31.58 \%$ and $81.58 \%$, respectively, which reflects the sensitivity of the chemotherapy regime and predicts patient prognosis $(20,21)$.
The overall median PFS time was 6.5 months. The median PFS for patients receiving the combination therapy as a first-line treatment was 7.5 months in TNBC, which is similar to the figure reported by $\mathrm{Hu}$ et al. (22) of a median PFS of 7.73 months for a therapy of a gemcitabine combined with cisplatin; however, the gastrointestinal reaction tolerance of patients was better in the present study. By the end of our follow-up studies, the median PFS time was still 6 months for patients receiving the treatment as third-line or above therapy, which demonstrates its efficacy for patients who have been treated with multilines of chemotherapy. The preliminary results of this study showed that capecitabine in combination with lobaplatin has a relatively high DCR and PFS and causes less toxic side effects in MBC patients. It is worth noting that since our data were based on a retrospective single-arm study, the small sample size and the lack of a comparison arm might reduce the reliability of the results. Additionally, these results might also be influenced by biases in clinical reasoning and judgment.

We also evaluated the side effects of the combination therapy. Myelosuppression is the main toxicity of lobaplatin observed in previous trials $(23,24)$, and thrombocytopenia is the most common dose-limiting toxicity. In the current study, the main adverse reactions were hematological toxicity, which manifested as neutropenia, anemia, and thrombocytopenia, and the occurrence of grade $3 / 4$ myelosuppression was $31.58 \%$. Another common side effect was gastrointestinal reactions; the occurrence of grade $3 / 4$ was $10.53 \%$. All patients recovered from the side effects after symptomatic treatment with the exception of 2 patients, who stopped the treatment due to the toxicity. Increased samples sizes could identify the rate of intolerable toxicity more accurately in patients receiving the combination therapy.

Further, we found that the prognosis outcome was related to the side effects. Specifically, we found significantly improved clinical outcomes in patients who experienced myelosuppression and/or gastrointestinal reactions. In previously published research, strong evidence has also been found of an association between improved clinical outcomes, including OS and DFS, and chemotherapyinduced myelotoxicity $(25,26)$. The mechanism underlying the association between chemotherapyinduced myelosuppression and clinical outcome is unclear; however, it may be involved in the control of the microenvironment in sites of metastatic spread $(27,28)$, and it may be correlated to the biological driver of the disease 


\section{Page 8 of 9}

directly (29). This was a single-arm retrospective study, and it was not a randomized controlled study. There was not enough number of cases of patients received either capecitabine or lobaplatin as single therapy in our institute, therefore, no comparison between the single therapy with the combined therapy of capecitabine and lobaplatin was performed.

In conclusion, capecitabine in combination with lobaplatin in treating advanced HER-2 negative BC showed good efficacy and tolerability, which provides strong evidence to support the application of this combination therapy as a beneficial treatment option for MBC patients.

This combination therapy has not been widely used in the clinic. This was the first study to report the efficacy and safety of the combination therapy of capecitabine and lobaplatin. At present, our sample size is relatively small, and the follow-up time is short. In the later stage, we will expand the sample size, extend the follow-up time, and provide more evidence-based evidence for the clinical application of this combination therapy for patients in the future.

\section{Conclusions}

Lobaplatin and capecitabine combination therapy in advanced MBC patients is effective and well tolerated.

\section{Acknowledgments}

Funding: The project was supported by the National Youth Natural Science Foundation of China (Grant No. 81703548), the Jiangsu Provincial Medical Youth Talent (QNRC2016647), and the talents program of Jiangsu Cancer Hospital (2017YYCJH-13).

\section{Footnote}

Reporting Checklist: The authors have completed the STROBE reporting checklist. Available at https:// dx.doi.org/10.21037/atm-21-2702

Data Sharing Statement: Available at https://dx.doi. org/10.21037/atm-21-2702

Conflicts of Interest: All authors have completed the ICMJE uniform disclosure form (available at https://dx.doi. org/10.21037/atm-21-2702). The authors have no conflicts of interest to declare.

\section{Yuan et al. Capecitabine and lobaplatin in advanced breast cancer}

Ethical Statement: The authors are accountable for all aspects of the work in ensuring that questions related to the accuracy or integrity of any part of the work are appropriately investigated and resolved. All procedures performed in this study involving human participants were in accordance with the Declaration of Helsinki (as revised in 2013). The study was approved by ethics board of Jiangsu Cancer Hospital (No. 2020-041). Individual consent for this retrospective analysis was waived.

Open Access Statement: This is an Open Access article distributed in accordance with the Creative Commons Attribution-NonCommercial-NoDerivs 4.0 International License (CC BY-NC-ND 4.0), which permits the noncommercial replication and distribution of the article with the strict proviso that no changes or edits are made and the original work is properly cited (including links to both the formal publication through the relevant DOI and the license). See: https://creativecommons.org/licenses/by-nc$\mathrm{nd} / 4.0 /$.

\section{References}

1. Grantzau T, Overgaard J. Risk of second non-breast cancer after radiotherapy for breast cancer: A systematic review and meta-analysis of 762,468 patients. Radiother Oncol 2015;114:56-65.

2. Gong DH, Ge JY, Chen YY, et al. HER2 overexpression in ductal carcinoma in situ is associated with ipsilateral breast cancer recurrence after conservative surgery. Transl Cancer Res 2020;9:3787-93.

3. Figueroa-Magalhães MC, Jelovac D, Connolly R, et al. Treatment of HER2-positive breast cancer. Breast 2014;23:128-36.

4. Ye X, Luo X, Du Q, et al. Efficacy and safety of lapatinib in Chinese breast cancer patients: a real-world study. Ann Transl Med 2020;8:240.

5. Heinemann V. Gemcitabine plus cisplatin for the treatment of metastatic breast cancer. Clin Breast Cancer 2002;3:24-9.

6. Egger SJ, Willson ML, Morgan J, et al. Platinumcontaining regimens for metastatic breast cancer. Cochrane Database Syst Rev 2017;6:CD003374.

7. Yang L, Qin S. Progression of lobaplatin as the third generation platinum drug. (In Chinese). Lin Chuang Zhong Liu Xue Za Zhi 2009;14:1134-9 .

8. Wu X, Tang P, Li S,et al. A randomized and open-label phase II trial reports the efficacy of neoadjuvant lobaplatin 
in breast cancer. Nat Commun 2018;9:832 .

9. Xie CY, Xu YP, Jin W, et al. Antitumor activity of lobaplatin alone or in combination with antitubulin agents in non-small-cell lung cancer. Anticancer Drugs 2012;23:698-705.

10. Wu Q, Qin SK, Teng FM, et al. Lobaplatin arrests cell cycle progression in human hepatocellular carcinoma cells. J Hematol Oncol 2010;3:43.

11. Eisenhauer EA, Therasse P, Bogaerts J, et al. New response evaluation criteria in solid tumours: revised RECIST guideline (version 1.1).Eur J Cancer 2009;45:228-47.

12. Shafei A, El-Bakly W, Sobhy A,et al. A review on the efficacy and toxicity of different doxorubicin nanoparticles for targeted therapy in metastatic breast cancer. Biomed Pharmacother 2017;95:1209-18.

13. Martín M. Platinum compounds in the treatment of advanced breast cancer. Clin Breast Cancer 2001;2:190-208.

14. Decatris MP, Sundar S, O'Byrne KJ. Platinum-based chemotherapy in metastatic breast cancer: current status. Cancer Treat Rev 2004;30:53-81.

15. Wang Z, Xu L, Wang H, et al. Lobaplatin-based regimens outperform cisplatin for metastatic breast cancer after anthracyclines and taxanes treatment. Saudi J Biol Sci 2018;25:909-16.

16. Wu Y, Xu XY, Yan F, et al. Retrospective study of the efficacy and toxicity of lobaplatin in combined chemotherapy for metastatic breast cancer. Onco Targets Ther 2019;12:4849-57.

17. Thomas ES, Gomez HL, Li RK, et al. Ixabepilone plus capecitabine for metastatic breast cancer progressing after anthracycline and taxane treatment. J Clin Oncol 2007;25:5210-7.

18. Massacesi C, La Cesa A, Marcucci F, et al. Capecitabine and mitomycin $\mathrm{C}$ is an effective combination for anthracycline-and taxane-resistant metastatic breast cancer. Oncology 2006;70:294-300.

19. Andres R, Mayordomo JI, Lara R, et al. Gemcitabine/ capecitabine in patients with metastatic breast cancer pretreated with anthracyclines and taxanes. Clin Breast Cancer 2005;6:158-62.

20. Robert NJ, Conkling PR, O'Rourke MA, et al. Results of a phase II study of pemetrexed as first-line chemotherapy in patients with advanced or metastatic breast cancer. Breast Cancer Res Treat 2011;126:101-8.

21. Vernieri C, Milano M, Mennitto A, et al. Antitumor activity and safety profile of weekly carboplatin plus paclitaxel in metastatic breast cancer: a ten-year, monocentric, retrospective study. Breast Cancer Res Treat 2017;165:365-73.

22. $\mathrm{Hu} \mathrm{XC}, \mathrm{Zhang} \mathrm{J}, \mathrm{Xu} \mathrm{BH}$, et al. Cisplatin plus gemcitabine versus paclitaxel plus gemcitabine as first-line therapy for metastatic triple-negative breast cancer (CBCSG006): a randomised, open-label, multicentre, phase 3 trial. Lancet Oncol 2015;16:436-46.

23. Guo W, Liao G, Gao H, et al. Randomized comparison of lobaplatin plus etoposide and cisplatin plus etoposide chemotherapy in patients with extensive-stage small cell lung cancer. Oncol Transl Med 2013;12:365-8.

24. Wang JQ, Wang T, Shi F, et al. A randomized controlled trial comparing clinical outcomes and toxicity of lobaplatin- versus cisplatin-based concurrent chemotherapy plus radiotherapy and high-dose-rate brachytherapy for FIGO stage II and III cervical cancer. Asian Pac J Cancer Prev 2015;16:5957-61.

25. Abraham JE, Hiller L, Dorung L, et al. A nested cohort study of 6,248 early breast cancer patients treated in neoadjuvant and adjuvant chemotherapy trials investigating the prognostic value of chemotherapy-related toxicities. BMC Med 2015;13:306.

26. Ma RM, Chen CZ, Zhang W, et al. Prognostic value of chemotherapy-induced neutropenia at the first cycle in invasive breast cancer. Medicine (Baltimore) 2016;95:e3240.

27. Houghton AM, Rzymkiewicz DM, Ji H, et al. Neutrophil elastase-mediated degradation of IRS-1 accelerates lung tumor growth. Nat Med 2010;16:219-23.

28. Nozawa H, Chiu C, Hanahan D. Infiltrating neutrophils mediate the initial angiogenic switch in a mouse model of multistage carcinogenesis. Proc Natl Acad Sci U S A 2006;103:12493-8.

29. Rini BI, Cohen DP, Lu DR, et al. Hypertension as a biomarker of efficacy in patients with metastatic renal cell carcinoma treated with sunitinib. J Natl Cancer Inst 2011;103:763-73.

(English Language Editor: L. Huleatt)

Cite this article as: Yuan Y, Zhang L, Zhang Z, Qian Y, Teng Y. A study of the efficacy and tolerability of capecitabine and lobaplatin in advanced HER-2 negative breast cancer patients. Ann Transl Med 2021;9(14):1151. doi: 10.21037/atm-21-2702 